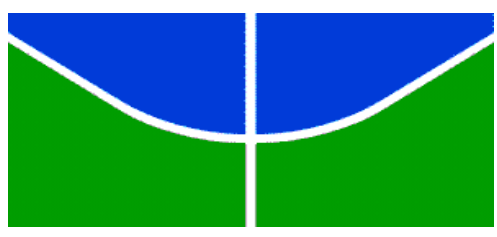

Universidade de Brasília

Centro de Estudos Avançados Multidisciplinares

Programa de Pós-Graduação em Direitos Humanos e Cidadania

A racialização dos(as) ciganos(as) e a experiência dos Calon de Belo Horizonte como sujeitos coletivos de um projeto histórico de liberdade

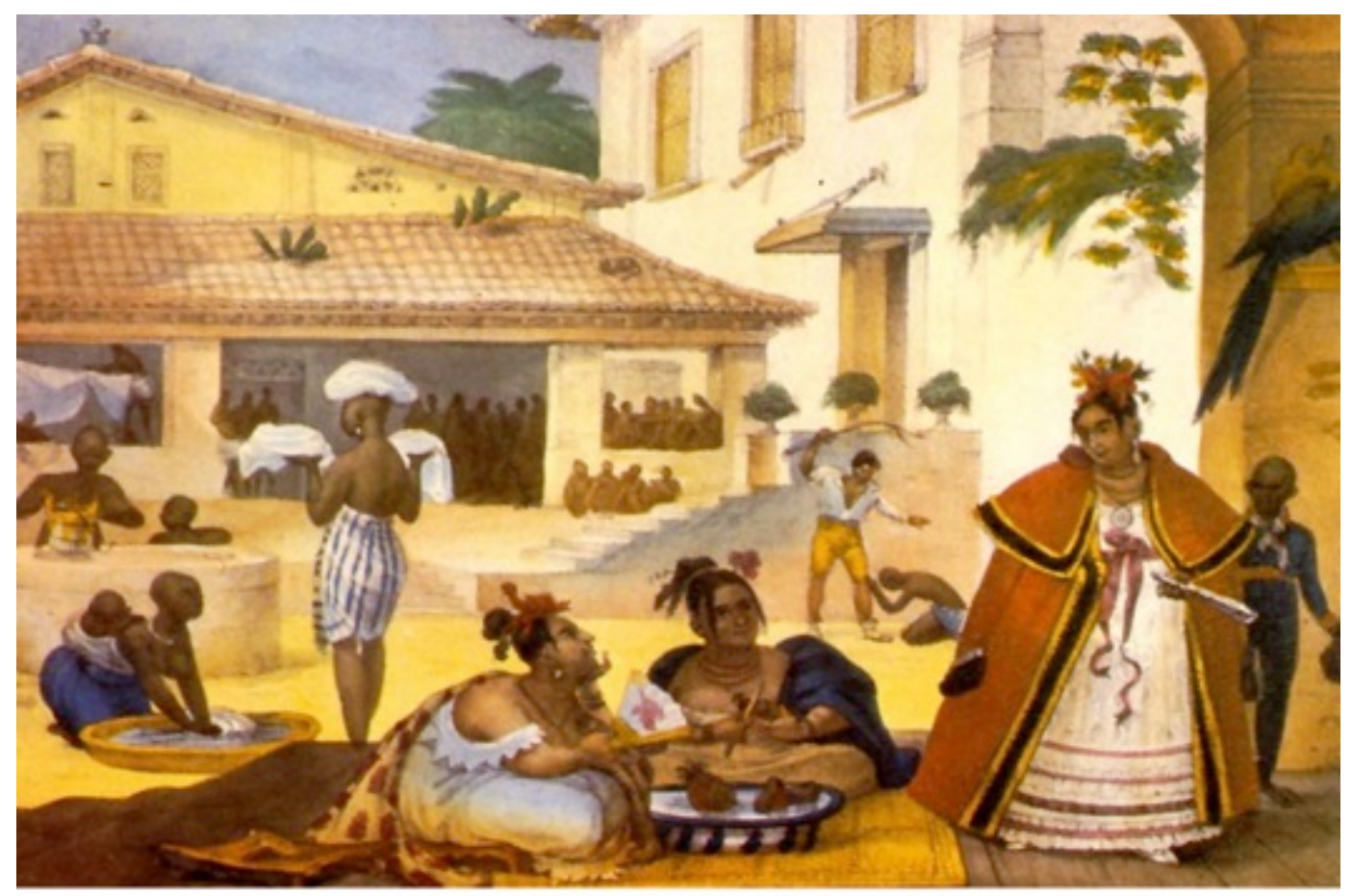

JEAN-BAP TS TE DEBRET (1768-1848): Interior de uma residéncia de ciganos, gravura do Tomo II Viagem Pitoresca e Histórica ao Brasil.

Priscila Paz Godoy 

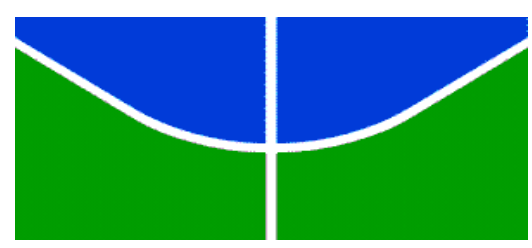

Universidade de Brasília

Centro de Estudos Avançados Multidisciplinares

Programa de Pós-Graduação em Direitos Humanos e Cidadania

\section{A racialização dos(as) ciganos(as) e a experiência dos Calon de Belo Horizonte como sujeitos coletivos de um projeto histórico de liberdade}

Priscila Paz Godoy

Dissertação apresentada ao Programa de Pós-Graduação em Direitos Humanos, da Universidade de Brasília - Área de Concentração: Direitos Humanos e Cidadania; Linha de Pesquisa: História, Direitos Humanos, Políticas Públicas e Cidadania -, como requisito parcial para obtenção do título de Mestre em Direitos Humanos.

Orientadora: Profa. Dra. Rita Laura Segato 
Ficha catalográfica elaborada automaticamente, com os dados fornecidos pelo(a) autor(a)

A racialização dos(as) ciganos(as) e a experiência dos Calon de Belo Horizonte como sujeitos coletivos de um projeto histórico de liberdade / Priscila Paz Godoy; orientador Rita Laura Segato. -- Brasília, 2015 .

$265 \mathrm{p}$.

Dissertação (Mestrado - Mestrado em Direitos Humanos e Cidadania) -- Universidade de Brasília, 2015 .

1. Raça. 2. Identidade. 3. Sujeitos Coletivos. 4. Pluralismo Histórico. 5. Direitos Humanos. I. Segato, Rita Laura, orient. II. Título. 

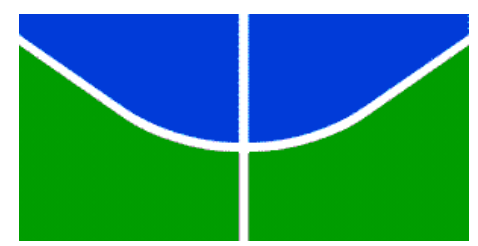

Universidade de Brasília

Centro de Estudos Avançados Multidisciplinares

Programa de Pós-Graduação em Direitos Humanos e Cidadania

Dissertação de autoria de Priscila Paz Godoy, intitulada "A racialização dos(as) ciganos(as) e a experiência dos Calon de Belo Horizonte como sujeitos coletivos de um projeto histórico de liberdade", submetida ao Programa de Pós-Graduação em Direitos Humanos e Cidadania - Área de Concentração: Direitos Humanos e Cidadania; Linha de Pesquisa: História, Direitos Humanos, Políticas Públicas e Cidadania -, como parte dos requisitos necessários para obtenção do grau de Mestre em Direitos Humanos, defendida e aprovada pela banca examinadora abaixo assinada:

\footnotetext{
Prof $^{\mathrm{a}}$. Dra . Rita Laura Segato (Presidente)

Universidade de Brasília - PPGDH/UnB

Prof $^{\mathrm{a}}$. Dr ${ }^{\mathrm{a}}$. Alejandra Pascual (Membro Externo)

Universidade de Brasília - FD/UnB

Prof $^{a}$. Dr ${ }^{\mathrm{a}}$. Cléria Botêlho da Costa (Membro Interno)

Universidade de Brasília - PPGDH/UnB

Prof ${ }^{a}$. Dra . Vanessa Maria de Castro (Suplente)

Universidade de Brasília - PPGDH/UnB
}

Brasília/DF, 2015 
Ao meu avô, José Paz (in memoriam) 


\section{AGRADECIMENTOS}

Escrever é, sobretudo, libertar-se, um exercício difícil de enfrentamento, de colocar-se no lugar do outro e de ver-se com o olhar do outro. Não à toa, o medo sempre me incomodou, porque é a expressão da ignorância e, por consequência, do preconceito. Estudar o racismo é e foi um grande desafio, instigado aqui pelos assim chamados "ciganos", a quem reservo toda a minha gratidão, gratidão de alguém, confesso, que já sentiu medo do "desconhecido".

Este estudo apresenta a ideia de raça, como um fenômeno puramente "mental", uma emergência histórica adversa a toda fixação biologista e a toda possibilidade de essencialismo. E propõe uma reflexão sobre o estereótipo nômade do povo cigano, que é associado diretamente à concepção biológica da "raça" e, para tanto, traz a experiência dos Calon de Belo Horizonte, Minas Gerais, como sujeitos coletivos que reinventam a sua própria história.

O tema do presente trabalho, inobstante a importância, ainda é pouco conhecido e, por isso, iniciei o percurso do estudo e da pesquisa na Universidade de Brasília. E para que ele se tornasse real contei com todo o entusiasmo, incentivo, cumplicidade e paciência, de Alvaro Larrabure Costa Correa, meu companheiro. Aqui não cabem os predicados.

Agradeço aos meus pais, Maria José Paz Godoy e José Geraldo da Silveira Godoy, por me ensinarem o que é empatia, coragem e perseverança; e à minha família, presente na alegria e na tristeza, na saúde e na doença, obrigada por todo o carinho.

As minhas avós materna e paterna (in memoriam), a minha gratidão pela grande inspiração de mulheres irreverentes e a frente de seus tempos. Ao meu avô materno (in memoriam), pela garra e luta por justiça.

Aos meus sogros, a quem sou grata pela atenção e pelo cuidado.

A Maria Antonia Lopes dos Santos, o meu obrigada pela paciência e ternura. 
À segunda turma do Programa de PósGraduação em Direitos Humanos e Cidadania da UnB (PPGDH/UnB): Bruna Junqueira Ribeiro, Diego Bernardo de Mendonça, Eliane Aparecida da Cruz, Érika Lula de Medeiros, Fábio Soares Alvino, Fredson Oliveira Carneiro, Irina Karla Bacci, Julia Barros Schirmer, Larissa da Silva Araújo, Raul Pietricovsky Cardoso, Vanessa Rodrigues de Araújo, Vitor Coelho Camargo de Melo e Viviane Fecher Moreira. Agradeço pela solidariedade, pelo acalanto nas inquietações e pela alegria.

Agradeço, em especial, aos(as) amigos(as) que tive a oportunidade de compartilhar as minhas agruras nos momentos mais difíceis, alegrias também! Ao Fredson Carneiro, pela inspiração poética e pelo carinho fraternal; A Zilda Pereira da Silva, pela paciência, disponibilidade e acolhida; a Larissa da Silva Araújo, por tudo e mais um pouco, a minha gratidão; A Viviane Fecher, pela serenidade, doçura e ternura; e a Diego Mendonça pela sensatez e paciência.

Às caras professoras Cléria Botelho da Costa e Vanessa Maria de Castro, cuja contribuição no aprendizado e cuja condução cuidadosa e carinhosa, na presente pesquisa, fez a diferença. Aos(às) professores(as) do PPGDH, pela possibilidade de trocas de conhecimento e saberes, pelo aprendizado e pelas provocações.

Minha irrestrita gratidão a Rita Laura Segato, professora e pesquisadora, que aceitou ao convite em me orientar na dissertação de mestrado do Programa de PósGraduação em Direitos Humanos e Cidadania, em um tema tão pouco conhecido. Toda a minha admiração e respeito pela ousadia, coragem e, sobretudo, por acreditar neste trabalho. Obrigada pelo estímulo na reflexão, que me permitiu pensar numa experiência real, cujo projeto de resistência é libertador, a dos Calon de São Gabriel.

Ao professor José Geraldo de Sousa Junior, agradeço a inspiração acadêmica, notadamente no que concerne ao Direito, não o dos livros, mas o real, o libertador e legítimo, porque proveniente de um processo de ressignificação constante de seus sujeitos coletivos.

Registro, aqui, o meu agradecimento carinhoso ao Professor Diogo Rosenthal Coutinho, sempre atento e solícito. 
Ao Procurador da República no Estado de Minas Gerais, Dr. Edmundo Antônio Dias Netto Júnior, pessoa vocacionada e comprometida para o exercício do seu ofício, meus profundos agradecimentos pela sensibilidade e disponibilidade sempre solidária e generosa para que este estudo se tornasse viável.

A minha gratidão a Yáskara Guelpa pelas inquietações, descobertas e ternura.

Aos ciganos Calon do bairro de São Gabriel, Belo Horizonte, Minas Gerais, meus agradecimentos especiais, pela disponibilidade, atenção e carinho.

A todos(as) os(as) assim chamados(as) ciganos(as) - a liberdade:

\author{
"...Liberdade, essa palavra \\ que o sonho humano alimenta \\ que não há ninguém que explique \\ e ninguém que não entenda..." \\ (Cecília Meireles)
}


"A tua raça de aventura quis ter a terra, o céu, o mar.

$\mathrm{Na}$ minha, há uma delícia obscura em não querer, em não ganhar...

A tua raça quer partir, guerrear, sofrer, vencer, voltar. A minha, não quer ir nem vir. A minha raça quer passar." (Cecília Meireles) 


\section{RESUMO}

Este estudo apresenta a ideia de raça, sob a perspectiva de Aníbal Quijano, que se define como um fenômeno puramente "mental", uma emergência histórica adversa a toda fixação biologista e a toda possibilidade de essencialismo. Propõe uma reflexão sobre o estereótipo nômade do povo cigano, que é associado diretamente à concepção biológica da "raça" e, para tanto, traz a experiência dos Calon de Belo Horizonte, Minas Gerais, como sujeitos coletivos que reinventam a sua própria história. Adota a categoria do "pluralismo histórico", formulada por Rita Segato que, no lugar da cultura como referencial da identidade fixa, coloca o projeto histórico de um povo como vetor central da diferença. E sustenta um diálogo entre a antropologia que, conforme Segato, se curva à demanda e à interpelação daquilo que outrora foi o seu "objeto", e os direitos humanos como processos históricos, conquistas e lutas por dignidade, bens e direitos, de acordo com as propostas de Roberto Lyra Filho, José Geraldo de Sousa Junior e Joaquín Herrera Flores.

Palavras-chave: Raça. Identidade. Sujeitos coletivos. Pluralismo histórico. Direitos humanos. 


\begin{abstract}
This study presents the idea of race from Anibal Quijano's perspective, defined as a purely "mental" phenomenon, a historical emergence contrary to the whole biologist fixation and to any possibility of essentialism. It proposes a reflection on the gypsy people's nomad stereotype directly associated to the biological concept of "race" and, with that purpose, brings forth the experience of the Calon people in Belo Horizonte, Minas Gerais, as collective subjects who have reinvented their own history. It adopts the "historical pluralism" category, formulated by Rita Segato, which, in lieu of the culture as a fixed identity referential, places the historical project of a people as the central difference vector. And it maintains a dialogue between the anthropology, which, according to Segato, yields to the demand and questioning of what was, in the past, its "object" and the human rights as historical processes, conquests and fights for dignity, possessions and rights, according to the propositions of Roberto Lyra Filho, José Geraldo de Sousa Junior and Joaquín Herrera Flores.
\end{abstract}

Keywords: Race. Identity. Collective subjects. Historical pluralism. Human rights. 


\section{LISTA DE ABREVIATURAS E SIGLAS}

$\S$ - Parágrafo

ACP - Ação Civil Pública

AGK - "Associação Guiemos Kalóns"

AGU - Advocacia Geral da União

ANEC - Associação Nacional das Etnias Ciganas

Art. - Artigo

$\mathrm{BH}$ - Belo Horizonte

CERCI - Centro de Estudos e Resgate da Cultura Cigana

CF - Constituição Federal

CJU - Consultoria Jurídica da União

CNPIR - Conselho Nacional de Políticas de Igualdade Racial

DNIT - Departamento Nacional de Infraestrutura e Transportes

DPU - Defensoria Pública da União

DPU-MG - Defensoria Pública da União de Minas Gerais

EMBRAPA - Empresa Brasileira de Pesquisa Agropecuária

IBGE - Instituto Brasileiro de Geografia e Estatística

ICP - Inquérito Civil Público

Inc. - Inciso

JK - Juscelino Kubitschek

MG - Minas Gerais

MPF - Ministério Público Federal

NuQ - Núcleo de Estudos de Populações Tradicionais e Quilombolas

OIT - Organização Internacional do Trabalho

ONU - Organização das Nações Unidas 
PRDC/MG - Procuradoria Regional dos Direitos do Cidadão

RFFSA - Rede Ferroviária Federal S/A

RMBH - Região Metropolitana de Belo Horizonte

n.p. - não paginado

SEDH - Secretaria Especial de Direitos Humanos da Presidência da República

SEPPIR - Secretaria de Promoção e Políticas de Igualdade Racial

SPU/MG - Superintendência do Patrimônio da União no estado de Minas Gerais

STF - Supremo Tribunal Federal

UFMG - Universidade Federal de Minas Gerais 


\section{LISTA DE FIGURAS}

Figura 1 - Memorial do Holocausto dos povos Sinti e Roma, em Berlim,

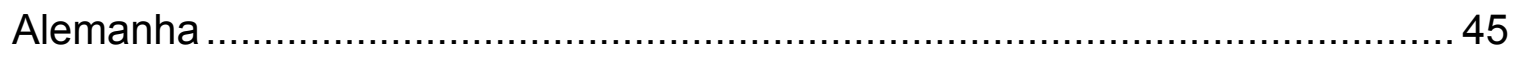

Figura 2 - Cronologia do genocídio dos Sinti e Roma ........................................... 46

Figura 3 - Monumento em homenagem aos Sinti e Roma, em Berlim, Alemanha (pedra triangular situada no meio do lago) ..... 


\section{SUMÁRIO}

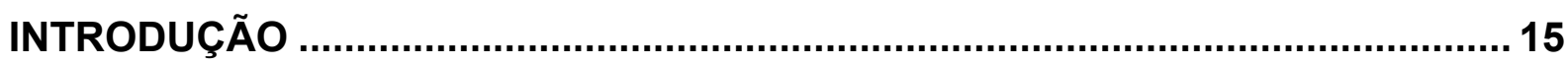

CAPÍTULO I - OS ASSIM CHAMADOS "CIGANOS" ............................................ 38

1.1 "Os Filhos do Sol" - uma origem controversa........................................... 38

$1.2 \mathrm{O}$ estereótipo racista de todo um povo ................................................... 40

1.3 Ciganos no Brasil: a liberdade como antinorma e afronta ao projeto

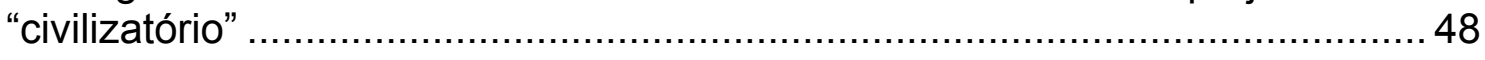

CAPÍTULO II - UMA LEITURA PERMANENTEMENTE HISTÓRICA .....................53

2.1 Raça e racismo no "moderno-sistema mundo" - a outra narrativa da

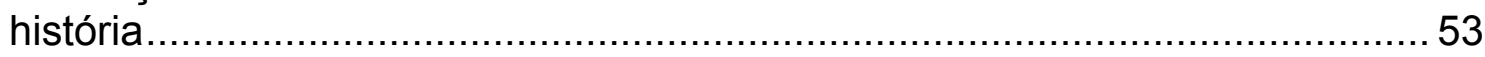

2.2. A emergência de identidades sociais historicamente novas e a

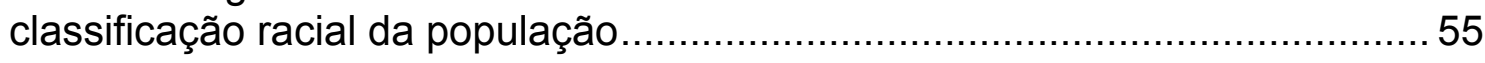

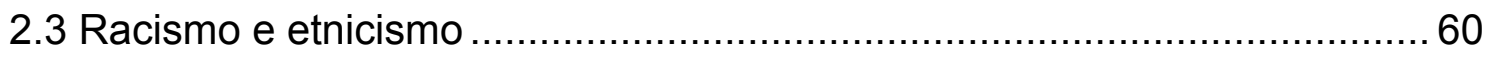

2.4 Palavras e conceitos - a contextualização permanente do pensamento e da

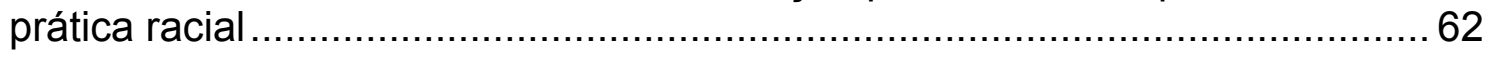

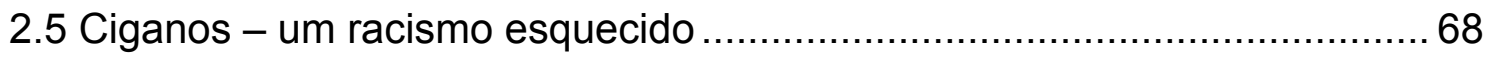

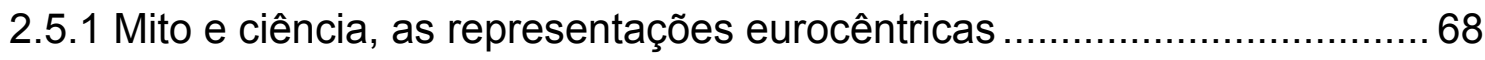

2.5.1.1. O trabalho indisciplinado no Brasil .................................................... 78

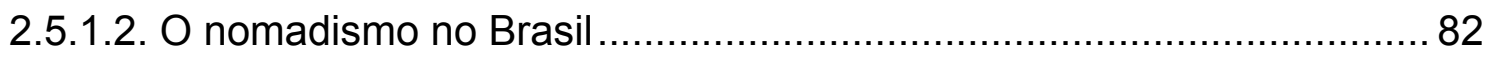

CAPÍTULO III - A EXPERIÊNCIA DOS CALON DO BAIRRO DE SÃO GABRIEL,

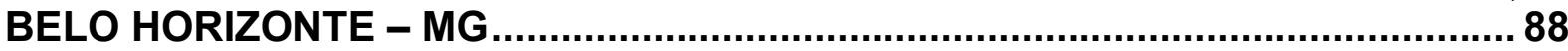

$3.1 \mathrm{O}$ início do processo de regularização fundiária, um breve histórico........... 88

3.2 Entre o movimento e o pouso - os Calon de São Gabriel ........................... 96

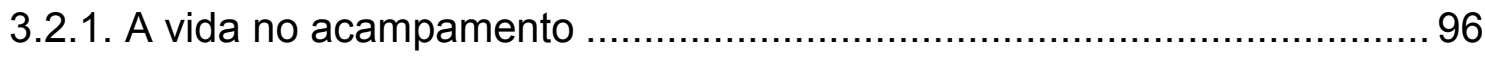

3.2.2 O pleito dos Calon de São Gabriel................................................... 100

CAPÍTULO IV - DA INVISIBILIDADE AO PROJETO HISTÓRICO DE

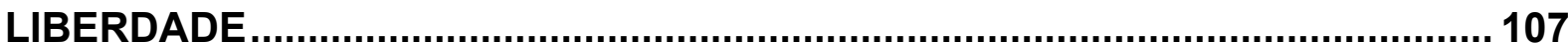

4.1 Entre a cultura e o pluralismo histórico - a resistência .............................. 107

4.2 Interculturalidade e Pluralismo Jurídico ................................................. 110

4.3 Entre a invisibilidade e a visibilidade - o "com-viver".............................. 122

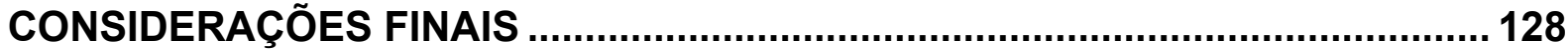

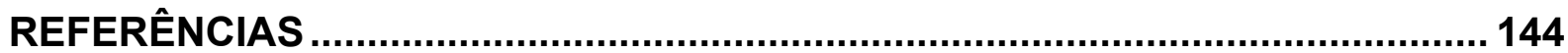

ANEXOS

ANEXO A 


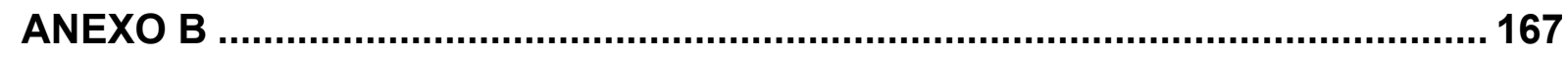

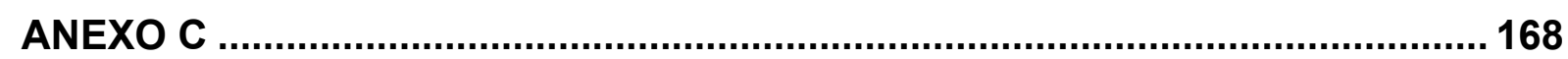

ANEXO D

ANEXO E

ANEXO F

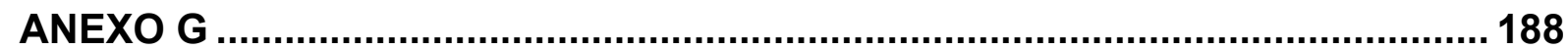

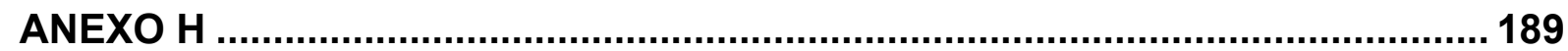

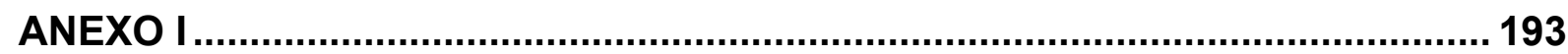

ANEXO J

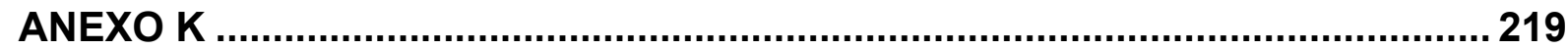

ANEXO L

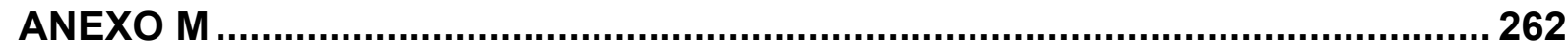




\section{REFERÊNCIAS}

AGUIAR, Roberto A. R. de. Os Filhos da flecha do vento. Pertinência e rupturas. Brasília: Letraviva, 2000.

APPELBAUM, N. P.; MACPHERSON, A. S. y ROSEMBLATT, K. A. "Introduction: racial nations. En: Nancy P. Appelbaum; Anne S. Macpherson y Karin Alejandra Rosemblatt (eds.). Race and nation in modern Latin America. Chapel Hill: The University of North Carolina Press, 2003.

ARIAS, E. e RESTREPO, J. Historizando raza: propuestas conceptuales y metodológicas. In: Crítica e Emancipación: Revista Latinoamericana de Ciencias Sociales. Año II, n. 3, Primer Semestre 2010, pp. 45-64.

BARTH, F. Towards Greater Naturalism in Conceptualizing Societies. In: Adam Kuper (Ed.). Conceptualizing Societies. London: Routledge, 1992. pp. 17-33.

BLOCH, J. Los gitanos. Buenos Aires: Editorial Universitária de Buenos Aires, 1962. pp. 6-61.

BLOCK, M. Moeurs et coutumes des Tziganes. Paris: Payot, 1936.

BORROW, G. Lavengro. London: John Murray, 1851.

BRASIL. Constituição (1988). Constituição da República Federativa do Brasil. Brasília: Senado Federal, 1988.

BRASIL. Decreto de 25 de maio de 2006. Institui o Dia Nacional do Cigano. Disponível em: http://www.planalto.gov.br/ccivil_03/_Ato20042006/2006/Dnn/Dnn10841.htm

BRASIL. Decreto n. 3.010, de 30 de março de 1999. Altera o art. $1^{\circ}$ do Decreto $n^{\circ}$ 2.661, de 8 de julho de 1998. Disponível em: http://www.planalto.gov.br/ccivil_03/decreto/D3010.htm

BRASIL. Decreto ${ }^{\circ} 5051$, de 19 de abril de 2004. Promulga a Convenção n. ${ }^{\circ} 169$ da Organização Internacional do Trabalho (OIT) sobre Povos Indígenas e Tribais. 
Disponível:

http://www.planalto.gov.br/ccivil_03/_ato2004-

2006/2004/decreto/d5051.htm

BRASIL. Decreto n. 6.040, de 7 de fevereiro de 2007. Institui a Política Nacional de Desenvolvimento Sustentável dos Povos e Comunidades Tradicionais. Disponível em: http://www.planalto.gov.br/ccivil_03/_ato2007-2010/2007/decreto/d6040.htm

BRASIL. Decreto n. ${ }^{\circ}$ 65.810, de 8 de dezembro de 1969. Promulga a Convenção Internacional sobre a Eliminação de todas as Formas de Discriminação Racial. Disponível

em:

http://legis.senado.gov.br/legislacao/ListaTextolntegral. action?id=94836

BRASIL. O Decreto $n^{\circ}$ 6.872, de 04 de junho de 2009. Aprova o Plano Nacional da Igualdade Racial, e institui o seu Comitê de Articulação e Monitoramento. Disponível em: http://www.planalto.gov.br/ccivil_03/_Ato2007-2010/2009/Decreto/D6872.htm

BRASIL. Lei n. 7.716, de 5 de janeiro de 1989. Define os crimes resultantes de preconceito de raça ou de cor. Disponível em: http://www.planalto.gov.br/ccivil_03/LEIS/L7716.htm

BRASIL. Lei n. 8.081, de 21 de setembro de 1990. Estabelece os crimes e as penas aplicáveis aos atos discriminatórios ou de preconceito de raça, cor, religião, etnia ou procedência nacional, praticados pelos meios de comunicação ou por publicação de

Disponível

em:

http://www.planalto.gov.br/ccivil_03/leis/L8081.htm.

BRASIL. Lei n. 11.483, de 31 de maio de 2007. Dispõe sobre a revitalização do setor ferroviário, altera dispositivos da Lei no 10.233, de 5 de junho de 2001, e dá outras providências. Disponível em: http://www.planalto.gov.br/ccivil_03/_ato20072010/2007/lei/l11483.htm

BRASIL. Ministério Público Federal. Resolução n. 6, de 16 de dezembro de 1993. Cria as Câmaras de Coordenação e Revisão no Ministério Público. Disponível em: http://bibliotecadigital.mpf.mp.br/bdmpf/bitstream/handle/123456789/70944/RES_CS MPF_1993_6.pdf?sequence=3\&isAllowed=y

BRASIL. PL 7774/2014. Dispõe sobre a inviolabilidade do domicílio da população cigana. Disponível em: http://www.camara.gov.br/proposicoesWeb/prop_mostrarintegra;jsessionid=9FDC7C 211405CC0DE6AE2EF703AEF9A3. proposicoesWeb2?codteor=1264018\&filename= $\mathrm{PL}+7774 / 2014$ 
BRASIL. PLS 248/2015 de 29/04/2015. Cria o Estatuto do Cigano. Disponível em: http://www12.senado.gov.br/ecidadania/visualizacaomateria?id=120952

BRASIL. Presidência da República. Secretaria de Políticas de Promoção da Igualdade Racial. Falta de políticas públicas para ciganos ainda é desafio para o governo. In: Agência Brasil, 24 de maio de 2011. Disponível em: http://www.seppir.gov.br/noticias/clipping-seppir/24-05.2011.

BRASIL. Secretaria de Políticas de Promoção da Igualdade Racial. Políticas de Promoção da Igualdade Racial. Disponível em: http://www.seppir.gov.br/apoiproj

BRASIL. Tribunal Regional Federal de Minas Gerais. Processo N ${ }^{\circ} 0001657-$ 29.2012.4.01.3803, da 19 ${ }^{a}$ Vara do estado de Minas Gerais. Belo Horizonte, 27 de junho de 2014. Disponível em: http://s.conjur.com.br/dl/dicionario-mostrar-definicaopejorativa.pdf

CABURÉ. Entrevista com os Calon do Bairro São Gabriel, Belo Horizonte/MG. Belo Horizonte/MG, 18 de setembro de 2014. Entrevista concedida a Priscila Godoy.

CARTA dos Superiores Provinciais da Companhia de Jesus da América Latina. $O$ Neoliberalismo na América Latina. São Paulo: Loyola, 1996.

CONVENÇÃO para a grafia dos nomes tribais. In: Revista de Antropologia, São Paulo, vol. 2, n² 2, 1954.

CURICACA. Entrevista com os Calon do Bairro São Gabriel, Belo Horizonte/MG. Belo Horizonte/MG, 18 de setembro de 2014. Entrevista concedida a Priscila Godoy.

CHINA, J. d'Oliveira. "Os ciganos do Brasil". In: Revista do Museu Paulista, Tomo XXI, 1936, pp. 323-669.

CLÉBERT, J-P. The Gypsies. London: Penguin, 1963.

COELHO, F. A. Os ciganos de Portugal: com um estudo sobre o calão. Lisboa: Dom Quixote, 1995.

COSTA, J. F. Ordem médica e norma familiar. Rio de Janeiro: Edições Graal, 1989. 
CORREAS, O (Org.). Pluralismo Jurídico: otros horizontes. Cidade do México: UNAM, 2007.

CORTÁZAR, J. Histórias de Cronópios e de Famas. Rio de Janeiro: Civilização Brasileira, 1994.

DEBRET, J. B. Viagem pitoresca e histórica ao Brasil. São Paulo: Martins / Brasília: INL, 1975.

DE LA CADENA, M. Introducción. In: Marisol de la Cadena (ed.). Formaciones de indianidad. Articulaciones raciales, mestizaje y nación en América Latina. Popayán: Envión, 2007.

DONOVAN, B. "Changing perceptions of social deviance: gypsies in early modern Portugal and Brazil". In: Journal of social History, vol. 26, 1992.

DORNAS FILHO, J. "Os ciganos em Minas Gerais". In: Rev. do Inst. Histórico e Geográfico de Minas Gerais, ano III, vol. III, 1948, pp. 138-187.

EINADI, B. e RIFAAT, A. "Nômades: uma liberdade vigiada". In: O Correio da UNESCO. Rio de Janeiro, ano 23, n 1, 1995.

ELIAS COSTA. União doa terras a ciganos da etnia Calom do Distrito Federal. In: Correio Braziliense, 24 de junho de 2015. Disponível em: http://www.correiobraziliense.com.br/app/noticia/cidades/2015/06/24/interna_cidadesdf,4877 77/uniao-doa-terras-a-ciganos-da-etnia-calom-do-distrito-federal.shtml

FAJARDO, R. Z. I. Um nuevo marco para la vigência y desarollo democrático de la pluralidade cultural y jurídica: constituición, juridicción indígena y derecho consuetidinario. Colombia, Peru y Bolivia. En: Desfaciendo Entuertos. Lima: Ceas, 1995.

FERRARI, Florência. O Mundo passa: uma etnografia dos Calon e suas relações com os seres brasileiros. Tese (Doutorado) - Departamento de Antropologia da Universidade de São, 2010.

FLORES, J. H. A (Re)invenção dos direitos humanos. Florianópolis: Fundação Boiteaux, 2009. 
FLORES, J. H. El vuelo de Anteo. Derechos humanos y crítica de la razón liberal. Bilbao: Editorial Desclée de Brouwer, 2000.

. De habitaciones propias y otros espacios negados. Uma teoria crítica de las opresiones patriarcales. Bilbao: Universidad de Deusto, 2005.

. Los derechos humanos como productos culturales. Crítica del humanismo abstracto. Catarata, 2005a.

. Introducción General. In: Marcelo Oliveira de Moura. Irrompendo no Real. Escritos de Teoria Crítica dos Direitos Humanos. Pelotas: Educat, 2005b.

La verdad de una Teoría Crítica de los Derechos Humanos. In: Marcelo Oliveira de Moura. Irrompendo no Real. Escritos de Teoria Crítica dos Direitos Humanos. Pelotas: Educat, 2005c.

FOTTA, M. A história do cigano nomadismo e a espacialidade Calon no Brasil. In: Nomadismo maneiras móveis de vida nas Américas, a Sociedade para a Continental e americano Estudos do Caribe. KONAK, Viena, Áustria, 2012.

FOUCAULT, M. Vigiar e punir: nascimento da prisão. Petrópolis: Vozes, 1977.

FRASER, A. História do Povo Cigano. Lisboa: Editorial Teorema, 1992.

FRASER, A. Los Gitanos. Barcelona: Ed. Ariel, 2005.

FRASER, A. The Gypsies. Oxford: Blackwell Publishers,1998.

GOFFMAN, E. Estigma. Trad. Márcia Bandeira de Mello Leite Nunes. Rio de Janeiro: Zahar Editores, 1975.

GOULD, S. J. A falsa medida do homem. 3. ed. São Paulo: Editora WMF Martins Fontes, 2014.

GUATTARI, F. "Espaço e poder: a criação de territórios na cidade". In: Espaço \& Debates: Revista de Estudos Regionais e Urbanos. São Paulo, ano V, n 16, 1985. 
GUIMARÃES ROSA, J. Tutaméia (Terceiras Estórias). 6. edição. Rio de Janeiro: Nova Fronteira, 1985.

GUIMARAIS, M. T. S. O associativismo transnacional cigano: identidades, diásporas e territórios. 2012 Tese (Doutorado em Geografia) - Universidade de São Paulo, São Paulo, 2012.

HAGUETTE, Teresa Maria Frota. Metodologias qualitativas na Sociologia. 3.ed.rev. e atual. Petrópolis: Vozes, 1992.

HANCOCK, I. The Pariah Syndrome. Ann Harbor: Karoma Publishers, 1987.

HAUPT, G. Antiziganismus und Sozialarbeit. Elemente einer wissenschaftlichen Grundlegung, gezeigt an Beispielen aus Europa mit dem Schwerpunkt Rumänien. Berlin, 2006.

HOLANDA FERREIRA, A. B. de. Novo Aurélio século XXI: o dicionário da língua portuguesa. $3^{a}$ ed. Totalmente revista e ampliada. Rio de Janeiro: Nova Fronteira, 1999.

IANNI, O. A metamorfose da etnia em raça. In: Pro-Posições, Vol. 15, n. 1 (43), jan./abr., 2004.

IBGE. Pesquisa de Informações Básicas Municipais - MUNIC 2009. Disponível em: http://www.ibge.gov.br/home/estatistica/economia/perfilmunic/2009/munic2009.pdf.

KENRICK, D. e PUXON, G. The Destiny of Europe's Gypsies. Sussex University Press, London, 1972.

KYMLICKA, Will. Ciudadanía multicultural. Uma teoria liberal dos direitos das minorias. Trad. De Carne Castells Auleda. Buenos Aires: Paidós, 1996.

KYMLICKA, Wil e NORMAN, Wayne. El retorno del ciudadano. Uma revisión de la producción reciente em teoria de la ciudanía. In: Revista de estudios sobre el Estado y la sociedad. La Política. Ciudadanía. El debate contemporâneo. Ottava: Paidós, Octubre, 1997. 
LIÉGEOIS, J. P. Los Gitanos Fondo de Cultura Econômica México, 1998.

LIMA, D. et. al. Relatório Antropológico sobre o Grupo Cigano Calon do Bairro de São Gabriel, Belo Horizonte. MINAS GERAIS. Ministério Público Federal. Procuradoria Regional dos Direitos do Cidadão em Minas Gerais. ICP $n^{\circ}$ 122000001978/2012-18. Belo Horizonte/MG, 2011.

LIMA, D. et. al. Avaliação da Demanda de Ocupação dos Ciganos Calon no Bairro São Gabriel, Belo Horizonte, Minas Gerais, Brasil. MINAS GERAIS. Ministério Público Federal. Procuradoria Regional dos Direitos do Cidadão em Minas Gerais. ICP $n^{\circ}$ 122000001978/2012-18. Belo Horizonte/MG, 2013.

LYRA FILHO, Roberto. A Nova Escola Jurídica Brasileira. In: Revista notícia do direito brasileiro. Brasília, n. ${ }^{\circ}$, p. 497-507, 2000.

- Desordem e processo: estudos sobre o direito em homenagem a Roberto Lyra Filho. Porto Alegre: Sergio Antonio Fabris Editor, 1986.

. Humanismo Dialético. In: Direito e Avesso. Brasília, ano II, $\mathrm{n}^{\circ}$ 3, Jan/Jun, 1983.

. O Direito que se ensina errado. Brasília: Centro Acadêmico de Direito da UnB, 1980a.

. O que é Direito. São Paulo: Editora Brasiliense, s.d.

. Para um Direito sem dogmas. Porto Alegre: Fabris, 1980b.

. O que é direito. $12^{\mathrm{a}}$ edição. Coleção Primeiros Passos no 62. São Paulo: Brasiliense, 1982.

MARTINS, R. Merkel homenageia vítimas ciganas do holocausto. In: Globo, 24 de outubro de 2012. Disponível em: http://www.dn.pt/inicio/globo/interior.aspx?content id=2844783\&seccao=Europa

MATTOS, I. R. O tempo Saquarema: a formação do estado imperial. São Paulo: Hucitec, 1990. 
MELLO, M. F. Os ciganos no Brasil \& Cancioneiro dos ciganos. Belo Horizonte: Itatiaia, 1981.

MIGNOLO, W. "Colonialidad del poder y subalternidad". In: Ileana Rodríguez (ed.). Convergencia de tiempos. Estudios subalternos/contextos latinoamericanos. Ámsterdam: Rodipi, 2001.

MIGNOLO, W. La idea de América Latina. La herida colonial y la opción decolonial. Barcelona: Gedisa, 2005.

MINAS GERAIS. Ministério Público Federal. Procuradoria Regional dos Direitos do Cidadão em Minas Gerais. ICP $n^{\circ}$ 122000001978/2012-18. Belo Horizonte/MG, 2011.

MINAYO, M.C., de S. Pesquisa Social. Petrópolis. Vozes, 25ª. Edição, 2007.

MINISTÉRIO PÚBLICO FEDERAL. Nota Técnica n. ${ }^{\circ}$ 6, PFDC/CAM/EB n. ${ }^{\circ}$ 07/2013. Brasília, 06 de agosto de 2013a. Disponível em: http://6ccr.pgr.mpf.mp.br/atuacaodo-mpf/classificacao-tematica/docs_ciganos/nota-tecnica-pfdc-cam-eb-no-07-2013

MINISTÉRIO PÚBLICO FEDERAL. Procuradoria da República em Minas Gerais. Ciganos Calon recebem certidão que reconhece posse de imóvel ocupado há 30 anos. In: Imprensa, 19 de dezembro de 2013b. Disponível em: http://www.prmg.mpf.mp.br/imprensa/noticias/indios-ecomunidadestradicionais/ciganos-calon-recebem-certidao-que-reconhece-posse-deimovel-ocupado-ha-30-anos.

MINISTÉRIO PÚBLICO FEDERAL. Procuradoria da República em Minas Gerais. MPF/MG vai à Justiça para mudar verbete do Dicionário Houaiss. In: Notícias, 27 de fevereiro de 2012. Disponível em: http://noticias.pgr.mpf.mp.br/noticias/noticias-dosite/copy_of indios-e-minorias/mpf-mg-vai-a-justica-para-mudar-verbete-dodicionario-houaiss

MONTEIRO, N. de Góis (Coord.). "KUBITSCHEK, João Nepomuceno". In: Dicionário biográfico de Minas Gerais; período republicano, 1889-1991; vol. 1, Belo Horizonte, Assembléia Legislativa do Estado de Minas Gerais, 1994, p. 325. 87. 
MONTEIRO, N.de Góis (Coord.). "OLIVEIRA, Juscelino Kubitschek". In: Dicionário biográfico de Minas Gerais; período republicano, 1889-1991, vol. 2, Belo Horizonte, Assembléia Legislativa do Estado de Minas Gerais, 1994, p. 477.

MOONEN, F. Anticiganismo e Políticas Ciganas na Europa e no Brasil. Recife: Edição do autor, 2013.

MOTA, Ático Vilas-Boas. A Origem dos Filhos do Sol. O Povo Cigano no Brasil. Vol. I. In: Rádio Senado, 24 de março de 2011.

NIEMANN, S. Eine nomadische Kultur der Freiheit. Vom Traum der Tsiganologie. In: Wulf D. Hund (Hrsg.). Zigeunerbilder. Schnittmuster rassistischer Ideologie. Duisburg, 2000.

NOGUEIRA, O. "Preconceito racial de marca e preconceito racial de origem". In: Tempo Social, Revista de Sociologia da USP, Vol. 19, n. 1, nov., 2006, pp. 287-308.

NOGUEIRA, O. Tanto preto quanto branco: estudo de relações raciais. São Paulo: T. A. Queiroz, 1985.

OKELEY, J. The Traveller-Gypsies. In: "L'étude des Tsiganes: un défi aux hégémonies territoriales et institutionnelles en anthropologie", Études Tsiganes 2/1994, pp. 39-58.

OKELY, J. Gypsies Travelling in Southern England. In: Alcock, A., Taylor, B., Welton, J. (Ed.). The future of cultural minorities. London: Macmillan Press, 1979, pp.55-83.

PAZ, F. M. Na poética da história: a realização da utopia nacional oitocentista. Curitiba: Ed. da UFPR, 1996.

PEREIRA, C. da C. A Cultura e seu Povo. O Povo Cigano no Brasil. Vol. III. In: Rádio Senado, 24 de março de 2011.

PEREIRA, C. DA COSTA. "Gli zingari in Brasile", Lácio Drom, ano 26, nº 6, 1990, pp. 3-5.

PIASERE, L., CAMPIGOTTO, A. From Margutte to Cingar: The Archeology of an Image, in M. Salo (Ed.). 100 Years of Gypsy Studies, Gypsy Lore Society. North American Chapter: Maryland, 1990. 
QUEIRÓS, B. C. de. Ciganos. São Paulo: Editora Global, 2004.

QUIJANO, Aníbal. Colonialidad del poder y clasificación social. In: Journal of WorldSystems Research (Riverside, California), Vol. VI, № 2, 2000a.

QUIJANO, Aníbal. Colonialidad, poder, cultura y conocimiento en América Latina. In: MIGNOLO, Walter (comp.). Capitalismo y geopolítica del conocimiento. El eurocentrismo y la filosofía de la liberación en el debate intelectual contemporâneo. Buenos Aires: Ediciones del Signo, 2000b.

QUIJANO, Aníbal. El fantasma del desarrollo en América Latina. In: Revista Venezolana de Economía y Ciencias Sociales (Caracas), Vol. 6, № 2, mayo-agosto, 2000c.

QUIJANO, A. “¡Qué tal raza!”. En: Familia y cambio social. Lima: CECOSAM, 1999.

QUIJANO, A. "Raza", ëtnia" y "nacion" en mariategui: cuestiones abiertas. En: FORGUES, Roland (ed.). José Carlos Mariátegui y Europa. La otra cara del descubrimiento. Lima, Peru: Amauta, 1992.

QUIJANO, A. Colonialidad del poder, eurocentrismo y América Latina. En Edgardo Lander (comp.). La colonialidad del saber: eurocentrismo y ciencias sociales. Perspectivas Latinoamericanas. CLACSO, Consejo Latinoamericano de Ciencias Sociales. Buenos Aires, Argentina. Julio de 2000. Disponible en la World Wide Web: http://bibliotecavirtual.clacso.org.ar/ar/libros/lander/quijano.rtf

QUIJANO, A. Colonialidade do poder, eurocentrismo e América Latina. En: Edgardo Lander (org). A colonialidade do saber: eurocentrismo e ciências sociais. Perspectivas latino-americanas. Colección Sur Sur, CLACSO, Ciudad Autónoma de Buenos Aires, Argentina, setembro 2005. pp. 227-278. Disponible en la World Wide Web: http://bibliotecavirtual.clacso.org.ar/ar/libros/lander/pt/Quijano.rtf

QUIJANO, A. e WALLERSTEIN, I. "La Americanidad como concepto, o América en el moderno Sistema Mundial". En: Revista Internacional de Ciencias Sociales. América: 1492-1992. Vol. XLIV, nº 4, diciembre, 1992, pp. 584-591.

QUIJANO, A. Cuestiones y horizontes: de la dependencia histórico-estructural a la colonialidad/descolonialidad del poder / Aníbal Quijano; selección a cargo de Danilo 
Assis Clímaco; con prólogo de Danilo Assis Clímaco. - 1a ed. - Ciudad Autónoma de Buenos Aires: CLACSO, 2014.

RESTREPO, E. “Cuestiones de método: ‘eventualización' y 'problematización' en Foucault”. En: Tabula Rasa, n. 8, Enero/Junio, 2008, pp. 111-132.

REZENDE, D. F. "A identidade cigana e o efeito de "nomeação": deslocamento das representações numa teia de discursos mitológico-científicos e práticas sociais." In: Rev. Antropologia, Vol. 49, n. 2, São Paulo, Jul/Dec, 2006, pp. 689-729. Disponível em: http://www.scielo.br/pdf/ra/v49n2/07.pdf.

REZENDE, D. F. de A. Transnacionalismo e Etnicidade - A Contrução Simbólica do Romanesthàn (Nação Cigana). 2000. Dissertação (Mestrado) - Curso de Mestrado do Departamento de Sociologia e Antropologia da Faculdade de Filosofia e Ciências Humanas da Universidade Federal de Minas Gerais. Belo Horizonte/MG, 2000.

RÖVID, R. One-size-fits-all Roma? On the normative dilemas of the emerging European Roma policy. In: Romani Studies, Vol. 21, Number 1, June, 2011, pp. 1-22.

SAINT-HILAIRE, A. de. Viagem à província de São Paulo, Belo Horizonte, Itatiaia, I São Paulo, Edusp, 1976.

SANTOS, B. de S. (org.). Reconhecer para libertar: os caminhos do cosmopolitismo multicultural. Rio de Janeiro: Civilização Brasileira, 2013.

SCHOLZ, Roswitha. Homo Sacer e Os Ciganos. O Anticiganismo - Reflexões sobre uma variante essencial e por isso esquecida do racismo moderno. Tradução de Boaventura Antunes, Lumir Nahodil e Virgínia Freitas, 12/2007. http://obeco.planetaclix.pt/roswitha-scholz7.htm

SCHOLZ, R. Differenzen der Krise - Krise der Differenzen. Die neue Gesellschaftskritik im globalen Zeitalter und der Zusammenhang von "Rasse", Klasse, Geschlecht und postmoderner Individualisierung [Diferenças da Crise - Crise das Diferenças. A nova crítica social na era global e a conexão de raça, classe, sexo e individualização pós-moderna], Bad Honnef, 2005a.

SCHOLZ, R. Der Mai ist gekommen. Ideologische Verarbeitungsmuster der Krise in wertkritischen Kontexten [Maio Chegou. O padrão de processamento ideológico da crise nos contextos da crítica do valor]. In: Exit! Krise und Kritik der 
Warengesellschaft [Exit! Crise e Crítica da Sociedade das Mercadorias]. 2005, pp. $106-137$.

SCHWARCZ, L. M. Racismo no Brasil. 2. edição. São Paulo: Publifolha, 2013.

SCHWARCZ, L. M. Ser peça, ser coisa: definições e especificidades da escravidão no Brasil: In: Lilia Moritz Schwarcz e Letícia Vidor de Sousa Reis (Orgs.). Negras imagens: ensaios sobre cultura e escravidão no Brasil. São Paulo: EDUSP/Estação Ciência, 1996.

SEMPRINI, A. Multiculturalismo. Bauru: EDUSC, 1999.

SEGATO, R. L. "Gênero e colonialidade: em busca de chaves de leitura e de um vocabulário estratégico descolonial". In: e-cadernos CES, n. 18, 2012. Disponível em: http://eces.revues.org/1533.

SEGATO, R. L. "Los cauces profundos de la raza latinoamericana: una relectura del mestizaje." En: Crítica y Emancipación, Año II, n. 3, Primer semestre 2010, pp. 1144.

. "Que cada povo teça os fios da sua história. O pluralismo jurídico em diálogo didático com legisladores." In: Revista de Direito da Universidade de Brasília. Vol. 1, jan. - jun. de 2014a.

SEGATO, R. L. La Perspectiva de la Colonialidad del Poder y el giro descolonial. In: José Luis Coraggio y Jean-Louis Laville. Reinventar la izquierda en el siglo XXI: hacia un dialogo norte-sur. Los Polvorines: Universidad Nacional de General Sarmiento, 2014. Disponível em: http://biblioteca.clacso.edu.ar/clacso/se/20140918020441/ReinventarLalzquierda.pdf

SEGATO, R. La Nación y sus otros. Raza, etnicidad y diversidad religiosa en tiempos de Políticas de la Identidad. Prometeo, 2007.

SILVERMAN, Carol. Romani routes: cultural politics, Balkan music in diaspora. Oxford: Oxford University Press, 2012.

SOUSA JUNIOR, J. G. de. Direito como Liberdade: O Direito Achado na Rua. Experiências Populares Emancipatórias de Criação do Direito. 2008. Tese (Doutorado) - Programa de Pós-Graduação em Direito da Universidade de Brasília. Brasília/DF, 2008. 
SPINELLI, S. [poema sem título]. s/d. Disponível em: https://it.wikipedia.org/wiki/Santino_Spinelli\#cite_note-1

SPNOZA, V. Brasil, Terra de Fulanos. O Povo Cigano no Brasil. Vol. II. In: Rádio Senado, 24 de março de 2011.

STEWART, M. The Time of The Gypsies. Oxford: Westview Press, 1997.

STOCKING, G. W. "The turn-of-the-century concept of race". En: Modernism/Modernity, Vol. 1, n. 1., January, 1994.

SUPREMO TRIBUNAL FEDERAL. Habeas Corpus nº 82.424 - Diário da Justiça 19/03/2004. In: Jurisprudência, s/d. Disponível em: http://www2.stf.jus.br/portalStfInternacional/cms/verConteudo.php?sigla=portalStfJuri sprudencia_pt_br\&idConteudo $=185077 \&$ modo $=\mathrm{cms}$

TAYLOR, C. et al. Multiculturalismo. Lisboa: Instituto Piaget, s/d.

TUAN, Yi-Fu, Espaço e lugar: a perspectiva da experiência. São Paulo: Difel, 1982.

TAUNAY, A. Império do Brasil, Lei de $1^{\circ}$ de outubro de 1828. In: Taunay, A. d'Escragnolle. História administrativa do Brasil. vol. VII (Organização Política e Administrativa do Império Brasileiro). Brasília, DASP-Centro de Documentação e Informática, 1974, pp. 297-311.

TEIXEIRA, R. Corrêa. História dos ciganos no Brasil. Recife: Núcleo de Estudos Ciganos, 2008

TEIXEIRA, R. C. Correrias de ciganos pelo território mineiro (1808-1903), Belo Horizonte: UFMG, 1998.

TEIXEIRA, R. C. História dos ciganos no Brasil, Recife: DHNET, 2000.

TEIXEIRA, R. C. Ciganos em Minas Gerais: breve história. Belo Horizonte: Crisálida, 2007. 
TRIVIÑOS, A.N.S. Introdução à pesquisa em ciências sociais: a pesquisa qualitativa em educação. S. Paulo: Atlas, 2008.

TROUILLOT, M. Adieu, culture. A new duty arises. In: Global transformations. Anthropology and the modern world. Nueva York: Palgrave McMillan, 2003.

TUAN, Yi-Fu. Espaço e lugar: a perspectiva da experiência. São Paulo: Difel, 1982.

UNITED STATES HOLOCAUST MEMORIAL MUSEUM. Genocide of European Roma (Gypsies), 1939-1945. In: Holocaust Encyclopedia, September 27, 2012. Disponível em: http://www.ushmm.org/wlc/en/article.php?Moduleld=10005219

WADE, P. Race, nature and culture: An anthropological perspective. Londres: Pluto Press, 2002.

WALSH, C. Interculturalidad, Estado, Sociedad: Luchas (de)coloniais de nuestra época. Primeira edición: Universidad Andina Simón Bolívar, Ediciones Abya-Yala, Quito, marzo 2009.

WALLERSTEIN, I. El capitalismo histórico. México DF: Siglo XXI, 1998.

WARAT, Luis Alberto. A condição transmoderna: o desencanto na cultura jurídica. In: Revista Humanidades, Editora da Universidade de Brasília, Vol. 9, n. 2 (32), s.d.

WILLIAMS, P. "Os eternos viajantes". In: O Correio da UNESCO, novembro de 1994, pp. 21-24. Disponível na web http://unesdoc.unesco.org/images/0009/000983/098363so.pdf.

WILLEMS, W. \& LUCASSEN, L. 1990. The Church of Knowledge: representation of gypsies in dutch encyclopedias and their sources: 1724-1984. In: M. Salo (ed.). 100 Years of Gypsy Studies, Gypsy Lore Society, Publication no. 5, Maryland, 1990, pp.31-50.

WOLKMER, A. C. Introdução ao Pensamento Jurídico Crítico. 7. ed. São Paulo: Saraiva, 2009. 
WOLKMER, A. C. Pluralismo jurídico: nuevo marco emancipatório em América Latina. En: Mauricio Garcia Villegas y César A. Rodríguez (eds.). Derecho y Sociedad en America Latina: Um debate sobre los estúdios jurídicos críticos. Bogotá: Instituto Latinoamericano de Servicios Legales Alternativos-Universidad Nacional de Colombia, 2003, pp. 247-259.

YÁSKARA, M. G. Brasil, Terra de Fulanos. O Povo Cigano no Brasil. Vol. II. In: Rádio Senado, 24 de março de 2011. 